\title{
Original
}

\section{Chinese tree shrews as a primate experimental animal eligible for the study of alcoholic liver disease: characterization and confirmation by MRI}

\author{
Zhihai SHI ${ }^{1) *}$, Huijie XING ${ }^{2) *}$, Chunli $\mathrm{QI}^{2)}$, Meixia $\mathrm{FANG}^{2)}$, Jiangnan $\mathrm{FU}^{2)}$ and Xingwang $\mathrm{ZHANG}{ }^{3)}$ \\ 1) Institute of Animal Husbandry and Veterinary, Henan Academy of Agricultural Sciences, 116 Huayuan Road, Zhengzhou, \\ Henan Province 450008, P.R. China \\ 2) Institute of Laboratory Animals, Jinan University, 601 West Huangpu Avenue, Guangzhou, Guangdong Province 510632, \\ P.R. China \\ 3) Department of Pharmaceutics, School of Pharmacy, Jinan University, 601 West Huangpu Avenue, Guangzhou, Guangdong \\ Province 510632, P.R. China
}

\begin{abstract}
There has been a lack of suitable fatty liver models and characterization techniques for histopathological evaluation of alcoholic fatty liver (AFL). This work aimed to exploit an magnetic resonance imaging (MRI) technique for characterizing an alcohol-induced fatty liver model established in tree shrews (Tupaia belangeri chinese). The animals were treated with $15 \%$ alcohol for two weeks instead of drinking water to induce AFL. Blood alanine aminotransferase (ALT), aspartate aminotransferase (AST), alcohol, and liver malondialdehyde (MDA) concentrations were determined, and the histopathology of the liver was checked by hematoxylin \& eosin (HE) and Oil red $O$ staining on day 0 and on the 4 th, 7 th and 14 th days after alcohol feeding. MRI was used to trace the histopathological changes in the liver of tree shrews in real time. Compared with the control group, the levels of ALT, AST, and MDA significantly increased in the alcohol-induced group and were positively correlated with the induction time. HE and Oil red $O$ staining revealed that a moderate fatty lesion occurred in the liver on the 4 th day and that a serious AFL was successfully induced on the 14th day. MRI further confirmed the formation of AFL. MRI, as noninvasive examination technique, provides an alternative tool for accurate characterization of AFL in live subjects. It is comparable to $\mathrm{HE}$ or Oil red $\mathrm{O}$ staining for histopathological examination, but is more suitable by virtue of its high flexibility and compliance. The AFL model of tree shrews combined with MRI characterization can work as a platform for studying fatty liver diseases and medications for their treatment.

Key words: alcohol induction, alcoholic fatty liver, histopathological check, magnetic resonance imaging, tree shrews
\end{abstract}

\section{Introduction}

Alcoholic liver disease (ALD) is a kind of alcoholtoxic hepatic disease resulting from chronic alcoholism, and it has a high incidence and mortality worldwide [9, 26]. Alcoholic fatty liver (AFL) disease is the early stage of ALD, which is reversible and benign in pathology. The probability of AFL disease has been found to be approximately $80 \%$ in heavy drinkers who consume over
$80 \mathrm{~g}$ of alcohol every day [8]. If not treated or drinking is not halted, AFL disease can progress to more serious and irreversible alcoholic hepatitis, hepatic fibrosis, hepatic cirrhosis, and finally hepatonecrosis [8, 19, 27]. Hepatic steatosis and fat accumulation are the main histopathological features of AFL [16, 30]. Presently, liver biopsy remains the golden standard for diagnosis and classification of hepatic steatosis in the clinic [22]. However, trauma, bleeding, infection, and organ injury

(Received 3 June 2019 / Accepted 2 September 2019 / Published online in J-STAGE 26 September 2019) Corresponding authors: X. Zhang.e-mail: zhangxw@jnu.edu.cn, J.Fu.e-mail: fujiangnan126@126.com *These authors contributed equally to this work. 
caused by puncture gravely impact its [6].

Among various diagnosis techniques, noninvasive approaches are strongly recommended to estimate the presence and severity of hepatic steatosis. These techniques include a biochemical assay, ultrasonography, computed tomography (CT), and magnetic resonance imaging (MRI). In cases of mild or moderate hepatic steatosis, the patients often do not feel any discomfort. They will not ask for a medical intervention until the levels of liver enzymes such as alanine aminotransferase (ALT) and aspartate aminotransferase (AST) rise significantly [2]. Furthermore, the enzyme levels are not completely parallel to the degree of damage of the liver [1]. Thus, a biochemical assay is only able to serve as an auxiliary diagnosis. Ultrasonography is the most frequently used means to assess hepatic steatosis, but only $62-77 \%$ of patients assessed with it can be successfully diagnosed with a fatty liver. Moreover, the technique is easily subjected to bias from technicians [10]. CT can convey an objective assessment of fatty deposition in the liver, but it exposes patients to radiation. In addition, CT possesses a low sensitivity (5-30\%) for fatty infiltration, causing it to miss some cases of moderate hepatic steatosis [17].

With the advance of imageology, MRI, as a noninvasive detection tool, has attracted more attention in qualitative and quantitative evaluation of fatty liver [21, $28,29]$. Compared with CT, MRI has dominant advantages based on its high resolution, use of multi-panel imaging, lack of radiation, and high accuracy with respect to hepatic fat content [18, 23]. Furthermore, it can provide a dynamic looking into the pathological changes of a liver suffering from steatosis [33]. Meanwhile, it has been shown that MRI quantification toward hepatic fat content is highly correlated with the outcomes of histopathological examination [11, 15]. Therefore, MRI is regarded as a more ideal detection technique for hepatic fat content that varies in fatty liver diseases. In a previous report, we established an AFL model of tree shrews (Tupaia belangeri chinese) and demonstrated it to be promising for insight into the pathology and medication for ALD [32]. However, the dynamic changes of hepatic fat content in the AFL model and pathologic process have not been well characterized.

In this study, we reconstructed the AFL model of tree shrews by means of alcohol feeding and monitored the hepatic fat content of the model in real time with MRI based on T2-weighted imaging with or without fat saturation. The liver injury model caused by a short-term alcohol induction is not only suitable for evaluation of acute hepatopathy but is also suitable for evaluation of chronic hepatopathy, since a similar pathogenesis such as hepatocyte damage, abnormal lipid metabolism, and massive lipid accumulation, take place in both cases. To verify the suitability of this model for hepatopathy study, hematological ALT, AST, and alcohol concentrations as well as hepatic malondialdehyde (MDA) were determined for model animals after alcohol induction. Hematoxylin \& eosin (HE) and Oil red O staining of liver tissues were performed to validate the reliability and effectiveness of the MRI technique in characterizing AFL.

\section{Materials and Methods}

\section{Materials}

Edible alcohol was obtained from Beijing Red Star Co., Ltd. (Beijing, China). ALT and AST detection kits were purchased from Roche (Shanghai, China). An MDA assay kit was provided by Nanjing Jiancheng Bioengineering Institute (Nanjing, China). Ethanol assay kit was supplied by BioAssay Systems (Hayward, CA, USA). Oil red $\mathrm{O}, \mathrm{HE}$ and other reagents were purchased from Sigma-Aldrich (Shanghai, China).

\section{Animals and alcohol induction}

Tree shrews (120-130 g, male and female in half) were provided by the experimental animal center of Kunming Medical College (Kunming, China). The animals were housed in an alternate light-dark cycle $(12 \mathrm{~h})$ room with a temperature of $22-26^{\circ} \mathrm{C}$ and relative humidity of $45-$ $75 \%$. Tree shrews were raised with a complete fodder and free access to water. They were allowed to acclimate themself to the environment for 10 days before alcohol feeding.

Animal experiments were carried out in accordance with the Guidelines issued by the Experimental Animal Ethical Committee of Jinan University. Tree shrews were continually fed $15 \%$ alcohol (v/v) for 14 days. The control group was given pure water other than food. At predetermined time points (day 0 and the 4 th, 7 th, and 14th days), four tree shrews were dislodged and subjected to MRI scanning for liver examination. Next, an appropriate amount of blood was sampled from the abdominal aorta of the tree shrews and used for later hematological examinations. After that, the livers of the tree shrews were immediately excised from their bodies following $\mathrm{CO}_{2}$ asphyxiation and stored in $10 \%$ formalin until use. During the experiment, the general conditions of animals such as mental state, appetite, and liver modality were investigated and recorded.

\section{Hematological examination}

The collected blood samples were centrifuged at 3,000 
rpm for $10 \mathrm{~min}$ to separate the serum. The serum was then analyzed by ALT and AST detection kits according to the instructions of the manufacturers using a biochemical analyzer (Cobas Integra 400 Plus, Roche, Rotkreuz, Switzerland). The blood alcohol concentration (BAC) was quantified by an ethanol assay kit (BioAssay Systems, Hayward, CA, USA). Each assay was performed in triplicate.

\section{Determination of MDA in the liver}

Liver tissues were homogenized with saline at a weight/volume ratio of $1 / 2$. The prepared homogenates were centrifuged at 5,000 rpm for $10 \mathrm{~min}$ to collect the supernatants. MDA concentration in the supernatants was determined using a commercially available MDA assay kit (Nanjing Jiancheng Bioengineering Institute, Nanjing, China) based on thiobarbituric acid (TBA) reactivity [20]. The procedure was carried out following the manufacturer's protocol.

\section{Hepatic histopathological examination}

$\mathrm{HE}$ and Oil red O staining were employed to check the histopathological alterations of the liver tissue. Briefly, liver tissues preserved in $10 \%$ formalin were dehydrated using a gradient procedure $(85 \%$ alcohol for $2 \mathrm{~h}$ and $95 \%$ alcohol for $2 \mathrm{~h}$, followed by anhydrous alcohol for $1 \mathrm{~h}$ ) and then processed into paraffin sections after deparaffinization with xylene. After being stained by HE or Oil red O, the liver histopathology of tree shrews was inspected and photographed using an Olympus imaging system (CX31, Olympus, Tokyo, Japan). Hepatic pathology was scored according to the severity of liver injury based on analyses of $\mathrm{HE}$ and Oil red $\mathrm{O}$ staining images.

\section{Characterization of hepatic steatosis using MRI}

To fully evaluate the formation of AFL, tree shrews were subjected to MRI scanning to image the liver after they were rendered unconscious with ketamine anesthesia. Animals were subjected to MRI on day 0 and the 4th, 7th, and 14th days. MRI experiments were performed using a Signa 1.5T MRI scanner (GE Medical systems, Fairfield, CT, USA) with a TR/TE of 2,000/87.7 $\mathrm{ms}$, slice thickness of $3 \mathrm{~mm}, 8 \times 8 \mathrm{~cm}$ field of view, and $256 \times 224$ matrix. To acquire high-resolution MRI images, a tree threw was placed within an 8-channel wrist coil, and signals were collected using the fast recovery fast spin-echo (FRFSE) T2-weighted imaging (T2WI) sequence with or without fat saturation. Liver fat contents in tree shrews were quantitatively analyzed using Advantage Workstation (ADW 4.4) based on the outcomes of MRI signal intensity. The following equation was used to calculate the hepatic fat fraction (HFF):

$$
H F F(\%)=\frac{S I_{T 2}-S I_{T 2 f s}}{2 S I_{T 2}} \times 100 \%
$$

where $S I_{T 2}$ and $S I_{T 2 f s}$ denote the signal intensity ratio of the liver to the muscle obtained using T2WI and T2weighted imaging with fat saturation (T2WI fs) sequences, respectively.

\section{Statistical analysis}

The hepatic fat fraction of tree shrews quantified by MRI was compared with synchronous data from pathological assessment following treatment for $0,4,7$, and 14 days. All data are shown as the mean $\pm \mathrm{SD}(\mathrm{n}=4)$. Analysis of variance (ANOVA) was carried out to judge the significance using SPSS 16.0 (SPSS inc., Chicago, IL, USA). A $P$ value $<0.05$ was considered to indicate a statistically significant difference.

\section{Results}

\section{General conditions}

The tree shrews were active and agile in climbing and jumping and maintained a normal diet (no anorexia or reduced food intake) with glossy furs before induction. Their livers exhibited an ordinary modality with a smooth capsule and soft texture in the anatomical check (Fig. 1). However, the tree shrews showed effects such as mental suppression, drowsiness, and appetite loss after alcohol feeding. After induction for 4 days, the liver became red and blunt in morphology with reduced flexibility. On the 7th day, the liver swelled and appeared sallow along with capsule tautness, and the sections were fairly greasy (Fig. 1). At the end of the experiment, the liver had blunt edges; presented a dim, coarse appearance; and possessed a hard texture.

\section{Biochemical indices pertinent to AFL}

Serum ALT, AST, and BAC and hepatic MDA levels of tree shrews treated with $15 \%$ alcohol for different times are shown in Table 1. Compared with the levels on day 0 , the levels of ALT and AST in the blood markedly increased and the uptrend was positively correlated with the induction time. Tree shrews possessed an average $\mathrm{BAC}$ of $22.16 \pm 6.52 \mathrm{mg} / \mathrm{dl}$ before induction. On the 4th day, the BAC of alcohol-treated tree shrews increased to $54.26 \pm 9.38 \mathrm{mg} / \mathrm{dl}$ and was significantly higher than that on day 0 . However, the BAC of tree shrews then remained at around $72 \mathrm{mg} / \mathrm{dl}$. These results indicate that the tree shrews have developed a liver injury and had adapted to the alcoholic diet.

The level of MDA in the liver presented a change 


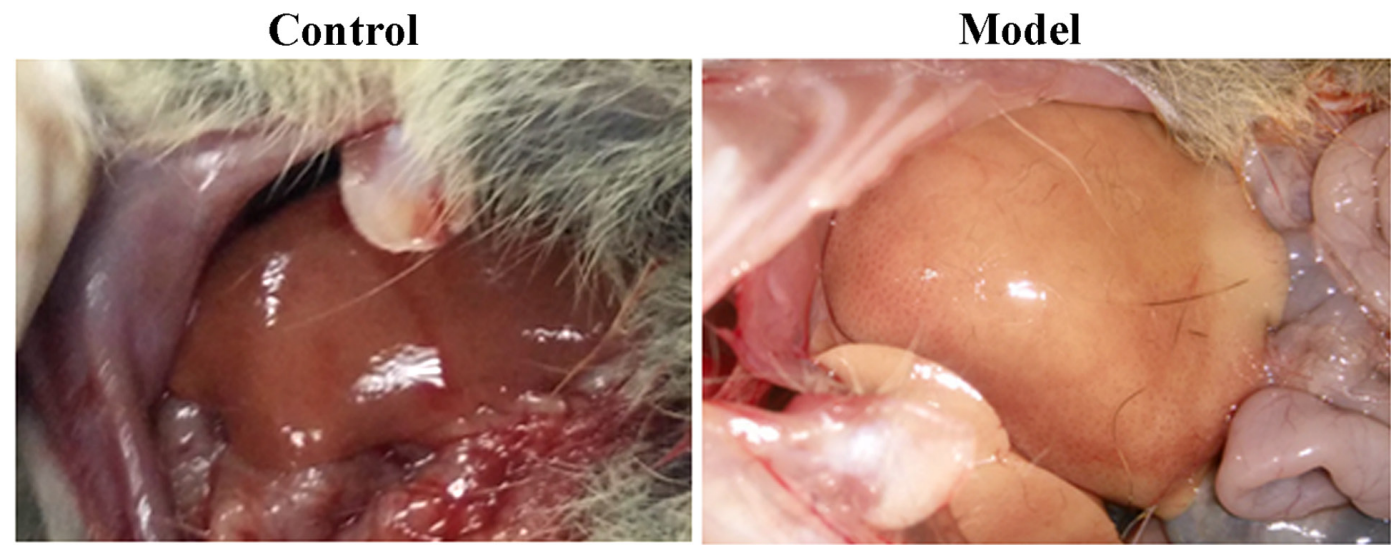

Fig. 1. Gross morphology of the liver of tree shrews before and 7 days after alcohol induction.

Table 1. Changes of biochemical indices in the blood and liver of tree shrews after feeding with water or $15 \%$ alcohol solution

\begin{tabular}{ccclc}
\hline \multirow{5}{*}{ Day } & \multicolumn{4}{c}{ Index } \\
\cline { 2 - 5 } & ALT (IU/L) & AST (IU/L) & BAC (mg/dl) & MDA (ng/mg tis.) \\
\hline 0 & $39.33 \pm 1.58$ & $179.83 \pm 39.64$ & $22.16 \pm 6.52$ & $0.12 \pm 0.03$ \\
4 & $118.83 \pm 32.25^{* *}$ & $228.44 \pm 53.13^{*}$ & $54.26 \pm 9.38^{* *}$ & $0.25 \pm 0.07^{* *}$ \\
7 & $169.66 \pm 42.41^{* *}$ & $316.83 \pm 60.85^{* *}$ & $70.31 \pm 8.65^{* *}$ & $0.33 \pm 0.06^{* *}$ \\
14 & $231.50 \pm 49.32^{* *}$ & $430.16 \pm 68.36^{* *}$ & $73.25 \pm 10.65^{* *}$ & $0.42 \pm 0.05^{* *}$ \\
\hline
\end{tabular}

Pared- $t$ test, $* P<0.05, * * P<0.01$ significantly different from the control. ALT, alanine aminotransferase; AST, aspartate aminotransferase; BAC, blood alcohol concentration; MDA, malondialdehyde.

similar to those of ALT and AST, which continuously increased with the time of alcohol feeding. The levels of hepatic MDA on the 4th day, 7 th and 14 th days were all significantly higher than that on day 0 . The degree of increase of MDA was proportional to the time of alcohol induction. Elevation of MDA in the liver suggested that alcohol-induced oxidative stress and lipid peroxidation were taking place in the tree shrews. Overall, the biochemical assay hinted that the tree shrews suffered from AFL after alcohol feeding.

\section{Liver histopathology}

HE staining micrographs of liver sections are shown in Fig. 2. Tree shrews that drank water exhibited a normal liver histopathology. The liver presented clear intercellular borders and integral hepatic lobules structurally with no obvious abnormality. On the 4 th day of alcohol induction, the hepatic lobules were roughly maintained, but hepatocyte swelling around the central vein appeared. Approximately 20-30\% of hepatocytes, based on an optical image analysis of the region of interest, showed cytoplasmic vacuolation, suggesting that a mild AFL was induced. Only small vacuoles (not more than $5 \mu \mathrm{m}$ ) appeared in the liver on the 4 th and 7 th days based on estimation from the scale bar. However, on the 14th day, there were also vacuoles that had grown to around $20 \mu \mathrm{m}$ in size (in addition to small ones). The vacuoles progressed until they were diffuse throughout the liver. After one week of induction, hepatic cord disarrangement, considerable endolysis, and more serious cytoplasmic vacuolation (60-80\% of hepatocyte aberrant) arose in the liver, showing a moderate AFL. On the 14th day, most hepatocytes showed hydropic swelling, hypochromatosis, or space-occupation by fat vacuoles, with about $70-90 \%$ of hepatocytes showing cytoplasmic vacuolation, demonstrating a serious AFL associated with tree shrews. HE staining revealed that hepatic steatosis in the tree shrews was aggravated as a function of induction time.

In order to further confirm the formation of AFL, Oil red $\mathrm{O}$ staining was performed to grade the fat accumulation of the liver. Figure 3 presents the typical histopathology of liver sections of tree shrews after induction for different times. There was no fat accumulation observable in the liver tissue of tree shrews treated with a normal diet. After 4 days of induction, liver sections exhibited regional red staining with pervasive fatty droplets (accounting for 20-30\% of total hepatocytes), which suggested the presence of mild fatty degeneration. The adipohepatic degree of the liver appeared more conspicuous, and $70-80 \%$ of hepatocytes were stained red after one week of induction, showing middle/severe fatty degeneration. On the 14th day of induction, almost all of hepatocytes ( $90 \%)$ were accompanied by a red- 

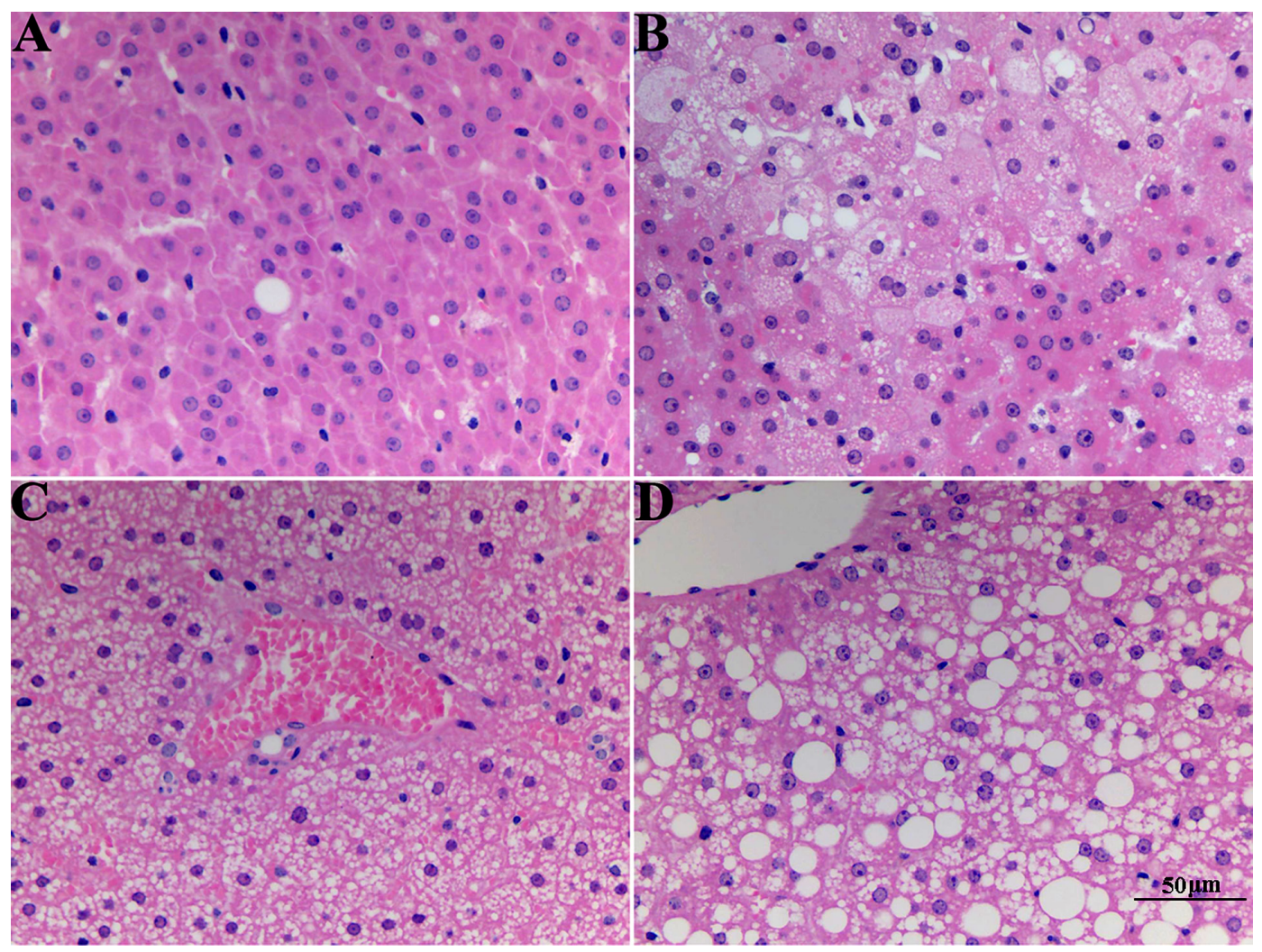

Fig. 2. Representative histological features of liver sections from tree shrews with hematoxylin \& eosin (HE) staining $(400 \times)$ inspected at different time points after induction: (A) normal control group, (B) model group on the 4th day, (C) model group on the 7th day, and (D) model group on the 14th day.
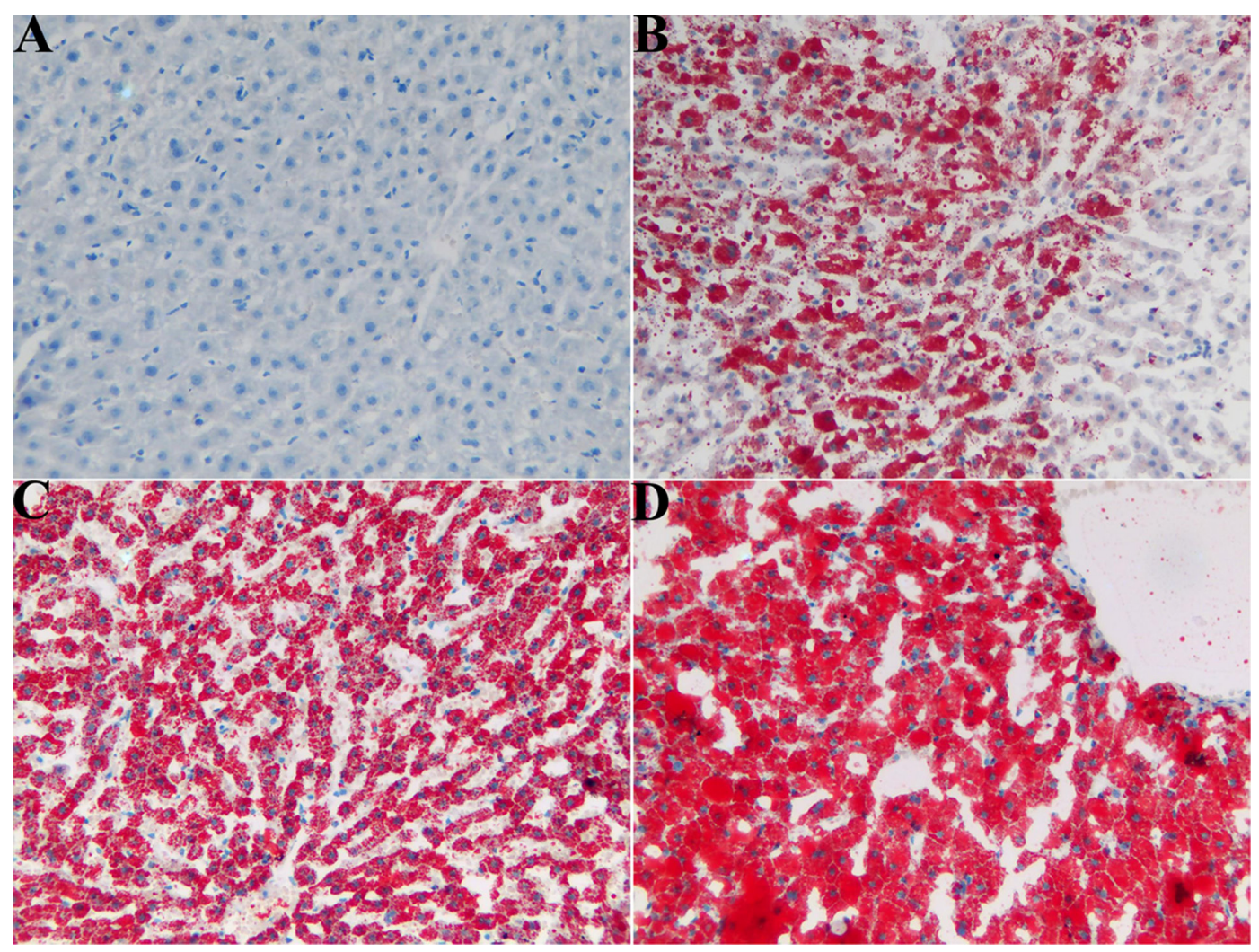

Fig. 3. Representative histological features of liver sections from tree shrews with Oil red O staining (200×) inspected at different time points after induction: (A) normal control group, (B) model group on the 4th day, (C) model group on the 7th day, and (D) model group on the 14th day. 
stained cytoplasm. Severe fatty degeneration had occurred in the livers of the tree shrews. These results are in agreement with those from HE staining. Taking the results of $\mathrm{HE}$ and Oil red $\mathrm{O}$ staining together, the liver injury in the tree shrews reached the grade of AFL, indicating that an AFL model can be successfully constructed in tree shrews by alcohol feeding.

Hepatic steatosis of the AFL model characterized by MRI

MRI images of livers of tree shrews on day 0 and the 4th, 7th, and 14th days of alcohol feeding are shown in Fig. 4. As seen from the images, the fat signals obtained using T2WI and T2WI fs sequences were fairly low for the control group (day 0 of alcohol feeding), indicating no fat accumulation in the liver. On the 4 th day, the fat signals were clearly displayed on the MRI image obtained using the T2WI sequence and could be more easily perceived through the pcolor. On the 7 th day, the liver fat signals markedly increased compared with that on the 4th day. This was a sign of aggravated hepatic steatosis, implying that AFL had begun to take shape in the tree shrews. When tree shrews were treated with $15 \%$ alcohol solution for 14 days, the fat signals of the liver were more apparent in terms of MRI images relative to the former groups. These results conveyed that more

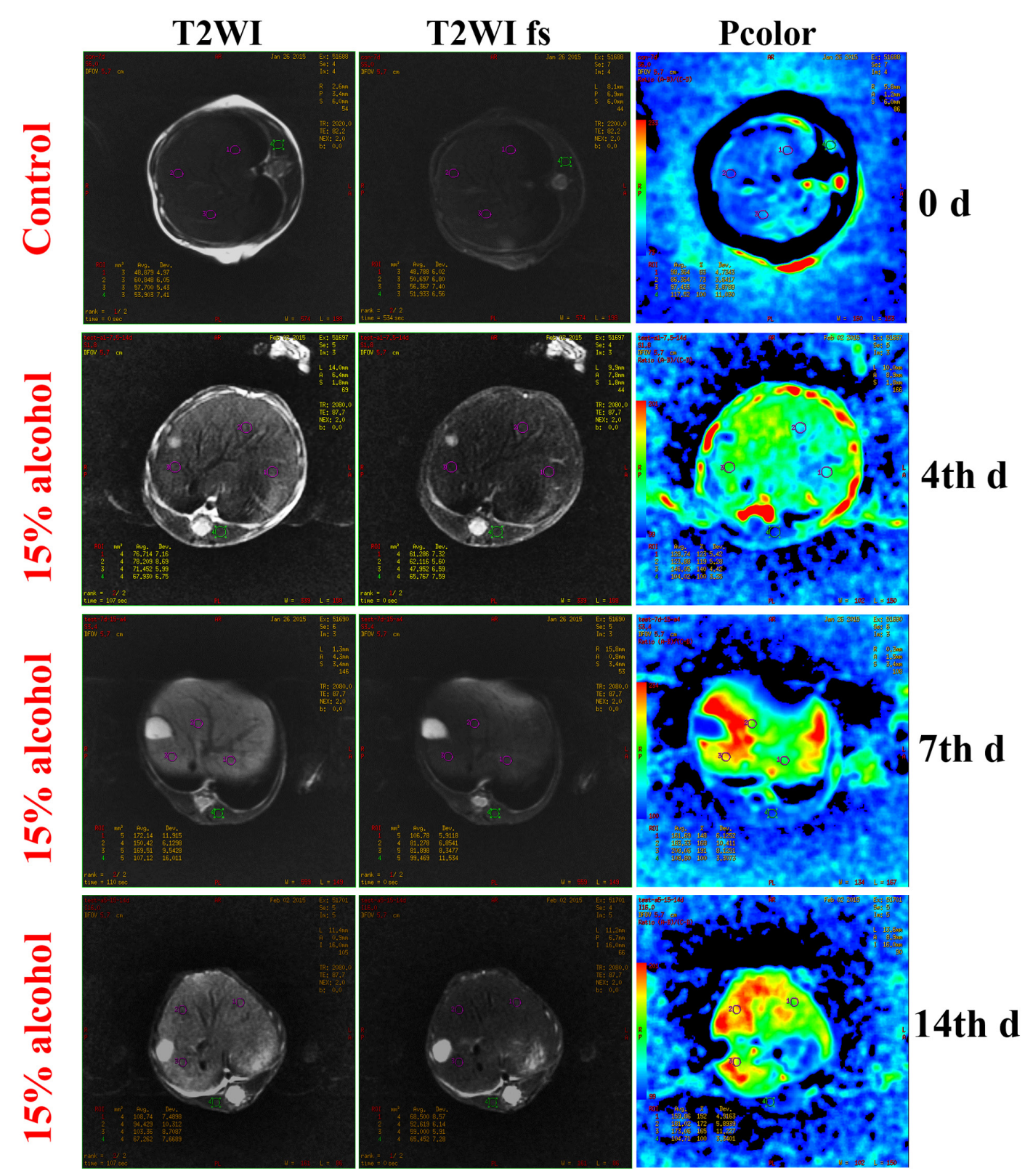

Fig. 4. Dynamic observation and quantitation of hepatic fat content using magnetic resonance imaging (MRI). MRI images of the liver were collected with T2-weighted imaging (T2WI) and T2-weighted imaging with fat saturation (T2WI fs) sequences, respectively. In the pcolor panels, the fat signal increases from blue to red gradually. 


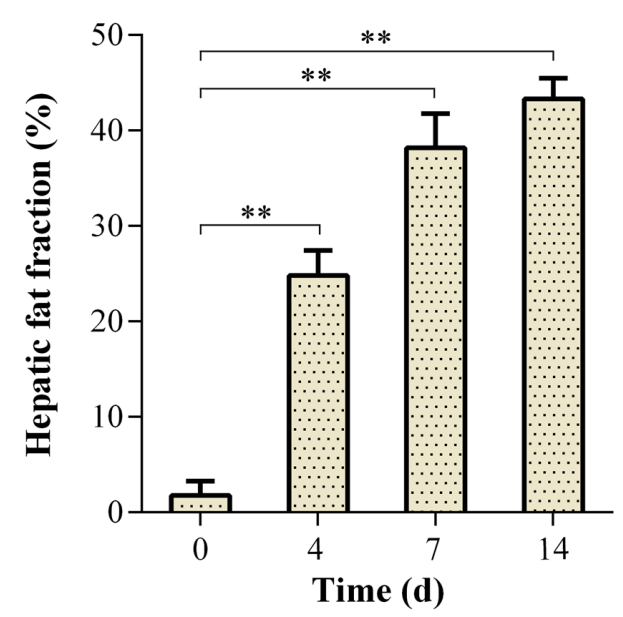

Fig. 5. Changes of hepatic fat fraction of tree shrews as a function of induction time. $* * P<0.01$ significantly different from the day 0 (Analysis of variance [ANOVA]).

serious hepatic steatosis was presented, indicating that the tree shrews had acquired a severe AFL.

The HFFs of tree shrews at different stages of induction were quantified by the differential fat signal intensity between the T2WI and T2WI fs sequences. The results are presented in Fig. 5. On the day 0, the tree shrews had an HFF of $1.76 \%$, which was a normal level of liver fat. However, HFF increased to $24.82 \%$ on the 4 th day of alcohol induction, indicating remarkable fat accumulation in the liver. On the 7 th and 14 th days, tree shrews had strikingly high HFFs that reached $38.1 \%$ and $43.3 \%$, respectively. It was shown that AFL could be properly induced in tree shrews with a short-term alcohol intervention. Generally, an HFF over 5\% is considered the clinical norm for diagnosis of fatty liver [13]. The qualitative and quantitative results of MRI with respect to hepatic steatosis were in agreement with those of the histopathological examination at the corresponding time points, demonstrating the suitability of MRI in characterization of the AFL model.

\section{Discussion}

Alcoholic liver disease is regarded as the most frequently diagnosed chronic liver disease other than viral hepatitis. As the health awareness of people has increased, AFL and its progressive diseases related to it have received more and more attention. As a result, there is strong desire for the application of more sensitive and acceptable techniques for early detection and diagnosis of AFL [4]. Although liver biopsy is useful as the golden standard for diagnosis of AFL, the technique is greatly limited because of its nondynamic characterization and invasiveness, which potentially causes issues, such as organ trauma, infection, and bleeding [25]. In contrast, a noninvasive technique is more suitable for assessment of temporal changes of AFL in terms of patient' compliance. Due to their low sensitivity and the presence of radioactivity, ultrasonography and CT techniques are sometimes difficult to meet the demands for an accurate and harmless diagnosis. MRI is undoubtedly the applicable method that best reflects the fatty infiltration in the liver. Currently, MRI based on the FRFSE T2WI sequence is extensively employed for quantitative evaluation of fatty liver [7, 24].

AFL can induce a spectrum of liver pathologies, from alcoholic steatohepatitis to fibrosis and cirrhosis, and can even induce liver failure [3]. T2WI and T2WI fs in MRI are simple and reproducible in terms of operation. The value for liver fat content determined by such techniques not only server as a diagnostic index of whether fatty liver is present or not but can also indicate the degree of hepatic steatosis. In this study, we utilized the FRFSE T2WI sequence with or without fat saturation to generate two different images. By importing the images into an ADW postprocessor, the average value of HFF could be calculated automatically by the software. The resulting HFF data were used to quantify the degree of hepatic steatosis in the AFL model.

The biochemical assay and histopathological examination confirmed the formation of fatty liver in the tree shrews. The HFF determined by MRI correlated well with the degree of hepatic steatosis estimated by HE and Oil red O staining. Quantitation of the liver fat content using MRI based on the signal difference of T2WI and T2WI fs sequences is relatively accurate but is less reported in the literature. The technique is able to comprehensively characterize the dynamic evolution of hepatic steatosis and simultaneously reduce the necessity for invasive liver biopsy and its associated complications.

An animal model mimicking human disease is an important tool for insight into the pathological mechanism of AFL diseases. There has been a lack of a suitable animal model for use in studying ALD. Tree shrews are a kind of lower primate animal species unique to the Chinese region and are characterized by a high-alcohol $\operatorname{diet}[5,31]$. In our previous study, we successfully constructed an AFL model based on tree shrews through continual feeding with a $20 \%$ alcohol solution for 14 days [32]. However, the model has a small shortcoming in that the animals tend to suffer from poor mental status, sometimes resulting in coma and death. This study reduced the alcohol concentration from $20 \%$ to $15 \%$ so as to properly induce AFL and improve the mental status of the animals. Likewise, 15\% alcohol feeding could induce a moderate to severe AFL, but no animals ap- 
peared coma and death. Our established model is more suitable for characterization of acute or chronic ALD. Other liver diseases share different pathologies that cannot be properly appreciated with this model. Currently, rats and mice are the commonly used experimental animals used for construction of an ALD model $[12,14]$. They are normally fed a Lieber-DeCarli ethanol liquid diet to induce ALD. However, Lieber-DeCarli feeding can only induce mild steatosis and marginal elevation of serum ALT, and it causes almost no inflammation in the liver. Compared with other ALD models, e.g., the GaoBinge model, our model does not require feeding of the animals an alcoholic diet by gavage. In addition, induction occurs naturally due to the alcohol diet of tree shrews and can be accomplished successfully in a short period. Considering the accessibility of model construction and the flexibility of MRI characterization, the AFL model based on tree shrews is highly promising as a research platform for understanding AFL-associated diseases.

\section{Conclusions}

Tree shrews characterized by a high alcoholic diet are an excellent fit as model animals used for the study of AFL diseases. An AFL model using tree shrews was constructed and confirmed by biochemical assay and histological examination in this study. The liver fat content was accurately quantified by MRI through T2WI and T2WI fs sequences. The technique was validated by histological examination and was shown to be rather practicable for characterization of the established AFL model, owing to its noninvasiveness, high sensitivity, and merit in dynamic monitoring. In short, this study involving AFL model and its characterization with MRI provides a useful tool for studying the pathological mechanism of AFL-associated diseases.

\section{Acknowledgment}

This work was supported by the Cultivation and Innovation Fund of Jinan University (11616319) and Independent Innovation Fund Project of the Henan Academy of Agricultural Science (2015ZZ31).

\section{References}

1. Adams, L.A. and Feldstein, A.E. 2011. Non-invasive diagnosis of nonalcoholic fatty liver and nonalcoholic steatohepatitis. J. Dig. Dis. 12: 10-16. [Medline] [CrossRef]

2. Baršić, N., Lerotić, I., Smirčić-Duvnjak, L., Tomašić, V. and Duvnjak, M. 2012. Overview and developments in noninvasive diagnosis of nonalcoholic fatty liver disease. World $J$. Gastroenterol. 18: 3945-3954. [Medline] [CrossRef]

3. Bruix, J., Gores, G.J. and Mazzaferro, V. 2014. Hepatocel- lular carcinoma: clinical frontiers and perspectives. Gut 63: 844-855. [Medline] [CrossRef]

4. Chiang, D.J. and McCullough, A.J. 2014. The impact of obesity and metabolic syndrome on alcoholic liver disease. Clin. Liver Dis. 18: 157-163. [Medline] [CrossRef]

5. Fan, Y., Huang, Z.Y., Cao, C.C., Chen, C.S., Chen, Y.X., Fan, D.D., He, J., Hou, H.L., Hu, L., Hu, X.T., Jiang, X.T., Lai, R., Lang, Y.S., Liang, B., Liao, S.G., Mu, D., Ma, Y.Y., Niu, Y.Y., Sun, X.Q., Xia, J.Q., Xiao, J., Xiong, Z.Q., Xu, L., Yang, L., Zhang, Y., Zhao, W., Zhao, X.D., Zheng, Y.T., Zhou, J.M., Zhu, Y.B., Zhang, G.J., Wang, J. and Yao, Y.G. 2013. Genome of the Chinese tree shrew. Nat. Commun. 4: 1426. [Medline] [CrossRef]

6. Fernández-Salazar, L., Velayos, B., Aller, R., Lozano, F., Garrote, J.A. and González, J.M. 2011. Percutaneous liver biopsy: patients' point of view. Scand. J. Gastroenterol. 46: 727-731. [Medline] [CrossRef]

7. Fischer, M.A., Nanz, D., Reiner, C.S., Montani, M., Breitenstein, S., Leschka, S., Alkadhi, H., Stolzmann, P., Marincek, B. and Scheffel, H. 2010. Diagnostic performance and accuracy of 3-D spoiled gradient-dual-echo MRI with water- and fat-signal separation in liver-fat quantification: comparison to liver biopsy. Invest. Radiol. 45: 465-470. [Medline] [CrossRef]

8. Gao, B. and Bataller, R. 2011. Alcoholic liver disease: pathogenesis and new therapeutic targets. Gastroenterology 141: 1572-1585. [Medline] [CrossRef]

9. Hartmann, P., Chen, W.C. and Schnabl, B. 2012. The intestinal microbiome and the leaky gut as therapeutic targets in alcoholic liver disease. Front. Physiol. 3: 402. [Medline] [CrossRef]

10. Hatta, T., Fujinaga, Y., Kadoya, M., Ueda, H., Murayama, H., Kurozumi, M., Ueda, K., Komatsu, M., Nagaya, T., Joshita, S., Kodama, R., Tanaka, E., Uehara, T., Sano, K. and Tanaka, N. 2010. Accurate and simple method for quantification of hepatic fat content using magnetic resonance imaging: a prospective study in biopsy-proven nonalcoholic fatty liver disease. J. Gastroenterol. 45: 1263-1271. [Medline] [CrossRef]

11. Hussain, H.K., Chenevert, T.L., Londy, F.J., Gulani, V., Swanson, S.D., McKenna, B.J., Appelman, H.D., Adusumilli, S., Greenson, J.K. and Conjeevaram, H.S. 2005. Hepatic fat fraction: MR imaging for quantitative measurement and display-early experience. Radiology 237: 1048-1055. [Medline] [CrossRef]

12. Kawaratani, H., Tsujimoto, T., Kitazawa, T., Yoshiji, H., Uemura, M. and Fukui, H. 2011. Therapeutic effects of cytokine modulator Y-40138 in the rat alcoholic liver disease model. $J$. Gastroenterol. Hepatol. 26: 775-783. [Medline] [CrossRef]

13. Khan, S.A., Wollaston-Hayden, E.E., Markowski, T.W., Higgins, L. and Mashek, D.G. 2015. Quantitative analysis of the murine lipid droplet-associated proteome during diet-induced hepatic steatosis. J. Lipid Res. 56: 2260-2272. [Medline] [CrossRef]

14. Ki, S.H., Park, O., Zheng, M., Morales-Ibanez, O., Kolls, J.K., Bataller, R. and Gao, B. 2010. Interleukin-22 treatment ameliorates alcoholic liver injury in a murine model of chronicbinge ethanol feeding: role of signal transducer and activator of transcription 3. Hepatology 52: 1291-1300. [Medline] [CrossRef]

15. Kim, S.H., Lee, J.M., Han, J.K., Lee, J.Y., Lee, K.H., Han, C.J., Jo, J.Y., Yi, N.J., Suh, K.S., Shin, K.S., Jo, S.Y. and Choi, B.I. 2006. Hepatic macrosteatosis: predicting appropriateness of liver donation by using MR imaging--correlation with histopathologic findings. Radiology 240: 116-129. [Medline] [CrossRef]

16. Lakshman, R., Shah, R., Reyes-Gordillo, K. and Varatharajalu, R. 2015. Synergy between NAFLD and AFLD and potential biomarkers. Clin. Res. Hepatol. Gastroenterol. 39:(Suppl 1): S29-S34. [Medline] [CrossRef]

17. Lall, C.G., Aisen, A.M., Bansal, N. and Sandrasegaran, K. 
2008. Nonalcoholic fatty liver disease. AJR Am. J. Roentgenol. 190: 993-1002. [Medline] [CrossRef]

18. Leporq, B., Lambert, S.A., Ronot, M., Boucenna, I., Colinart, P., Cauchy, F., Vilgrain, V., Paradis, V. and Van Beers, B.E. 2016. Hepatic fat fraction and visceral adipose tissue fatty acid composition in mice: Quantification with 7.0T MRI. Magn. Reson. Med. 76: 510-518. [Medline] [CrossRef]

19. Liu, J. 2014. Ethanol and liver: recent insights into the mechanisms of ethanol-induced fatty liver. World J. Gastroenterol. 20: 14672-14685. [Medline] [CrossRef]

20. Liu, L., Liu, Y., Cui, J., Liu, H., Liu, Y.B., Qiao, W.L., Sun, H. and Yan, C.D. 2013. Oxidative stress induces gastric submucosal arteriolar dysfunction in the elderly. World J. Gastroenterol. 19: 9439-9446. [Medline] [CrossRef]

21. Mehta, S.R., Thomas, E.L., Bell, J.D., Johnston, D.G. and Taylor-Robinson, S.D. 2008. Non-invasive means of measuring hepatic fat content. World J. Gastroenterol. 14: 34763483. [Medline] [CrossRef]

22. Paparo, F., Cenderello, G., Revelli, M., Bacigalupo, L., Rutigliani, M., Zefiro, D., Cevasco, L., Amico, M., Bandelloni, R., Cassola, G., Forni, G.L. and Rollandi, G.A. 2015. Diagnostic value of MRI proton density fat fraction for assessing liver steatosis in chronic viral C hepatitis. BioMed Res. Int. 2015: 758164. [Medline] [CrossRef]

23. Pavlides, M., Banerjee, R., Sellwood, J., Kelly, C.J., Robson, M.D., Booth, J.C., Collier, J., Neubauer, S. and Barnes, E. 2016. Multiparametric magnetic resonance imaging predicts clinical outcomes in patients with chronic liver disease. $J$. Hepatol. 64: 308-315. [Medline] [CrossRef]

24. Rastogi, R., Gupta, S., Garg, B., Vohra, S., Wadhawan, M. and Rastogi, H. 2016. Comparative accuracy of CT, dual-echo MRI and MR spectroscopy for preoperative liver fat quantification in living related liver donors. Indian J. Radiol. Imaging 26: 5-14. [Medline] [CrossRef]

25. Ravindran, S., Hancox, S.H. and Howlett, D.C. 2016. Liver biopsy: past, present and future. Br. J. Hosp. Med. (Lond.) 77 : 90-95. [Medline] [CrossRef]

26. Rehm, J., Samokhvalov, A.V. and Shield, K.D. 2013. Global burden of alcoholic liver diseases. J. Hepatol. 59: 160-168. [Medline] [CrossRef]

27. Schwartz, J.M. and Reinus, J.F. 2012. Prevalence and natural history of alcoholic liver disease. Clin. Liver Dis. 16: 659666. [Medline] [CrossRef]

28. Schwenzer, N.F., Springer, F., Schraml, C., Stefan, N., Machann, J. and Schick, F. 2009. Non-invasive assessment and quantification of liver steatosis by ultrasound, computed tomography and magnetic resonance. J. Hepatol. 51: 433-445. [Medline] [CrossRef]

29. Sijens, P.E. 2009. Parametric exploration of the liver by magnetic resonance methods. Eur. Radiol. 19: 2594-2607. [Medline] [CrossRef]

30. Springer, F., Machann, J., Claussen, C.D., Schick, F. and Schwenzer, N.F. 2010. Liver fat content determined by magnetic resonance imaging and spectroscopy. World J. Gastroenterol. 16: 1560-1566. [Medline] [CrossRef]

31. Wiens, F., Zitzmann, A., Lachance, M.A., Yegles, M., Pragst, F., Wurst, F.M., von Holst, D., Guan, S.L. and Spanagel, R. 2008. Chronic intake of fermented floral nectar by wild treeshrews. Proc. Natl. Acad. Sci. USA 105: 10426-10431. [Medline] [CrossRef]

32. Xing, H., Jia, K., He, J., Shi, C., Fang, M., Song, L., Zhang, P., Zhao, Y., Fu, J. and Li, S. 2015. Establishment of the tree shrew as an alcohol-induced Fatty liver model for the study of alcoholic liver diseases. PLoS One 10: e0128253. [Medline] [CrossRef]

33. Yamada, T., Obata, A., Kashiwagi, Y., Rokugawa, T., Matsushima, S., Hamada, T., Watabe, H. and Abe, K. 2016. GdEOB-DTPA-enhanced-MR imaging in the inflammation stage of nonalcoholic steatohepatitis (NASH) in mice. Magn. Reson. Imaging 34: 724-729. [Medline] [CrossRef] 